\title{
Ansiedad precompetitiva y motivación en nadadores costarricenses de élite
}

\author{
Haase, Mikaela \\ Ansiedad precompetitiva y motivación en nadadores costarricenses de élite \\ MHSalud, vol. 18, núm. 2, 2021 \\ Universidad Nacional, Costa Rica \\ Disponible en: https://www.redalyc.org/articulo.oa? $\mathrm{id}=237066090006$ \\ DOI: https://doi.org/10.15359/mhs.18-2.6
}

\section{(c) (1) 9}

Esta obra está bajo una Licencia Creative Commons Atribución-NoComercial-SinDerivar 3.0 Internacional. 


\title{
Ansiedad precompetitiva y motivación en nadadores costarricenses de élite
}

\author{
Precompetitive Anxiety and Motivation in Costa Rica Elite Swimmers \\ Ansiedade pré-competitiva e motivação em nadadores de elite da Costa Rica
}

\author{
Mikaela Haase \\ Universidad de Iberoamérica, Costa Rica \\ mikahaase1712@gmail.com \\ https://orcid.org/0000-0001-7483-0072
}

DOI: https://doi.org/10.15359/mhs.18-2.6

Redalyc: https://www.redalyc.org/articulo.oa?

id $=237066090006$

Recepción: 08 Junio 2020

Aprobación: 11 Febrero 2021

\section{RESUMEN:}

El objetivo de esta investigación es describir el manejo de la ansiedad precompetitiva en nadadores y nadadoras costarricenses de élite, entre los 14 y 22 años, identificando los elementos motivantes (externos e internos) y el cómo influye la relación de dichos atletas con su entrenador y con su grupo de pares en los procesos de motivación y ansiedad precompetitiva. Para ello se realizaron entrevistas semiestructuradas a finales del año 2018. Los resultados sugieren que entre los principales elementos asociados con mayores sensaciones de ansiedad se encuentran la falta de objetivos alcanzados antes de la competencia, el tener un bajo rendimiento durante los entrenamientos y estar al tanto de la importancia de la competición respectiva. Sus motivaciones se han clasificado en intrínsecas (competición olímpica, mejora de tiempos, ubicación en ránkings) y extrínsecas (familia, entrenador, equipo). Al existir una mala relación con el entrenador y su grupo de pares se pierde el interés de ir a entrenar y de competir. En conclusión, al presentar ansiedad precompetitiva, el rendimiento puede disminuir provocando un incumplimiento de objetivos, lo que lleva a que los atletas se empiecen a desmotivar, manteniendo la ansiedad para sus siguientes competiciones.

PALABRAS CLAVE: Ansiedad precompetitiva, motivación, natación, psicología deportiva.

\section{Abstract:}

This study aims to describe the management of precompetitive anxiety in elite Costa Rican swimmers aged 14 to 22 years old, identifying the motivating elements (external and internal) and how the relationship with their coach and their peer group influences the processes of motivation and precompetitive anxiety. Semi-structured interviews were conducted by the end of 2018. The results suggest that among the main elements associated with greater feelings of anxiety are the lack of objectives achieved before the competition, having a poor performance during training, and being aware of the importance of the respective competition. The elite swimmers' motivations have been classified as intrinsic (Olympic competition, time improvement, placement in rankings) and extrinsic (family, coach, team) ones. Finally, results suggest that a bad relationship with the coach and peer groups could result in a loss of interest in training and competing. In conclusion, when presenting precompetitive anxiety, performance can decrease, causing non-fulfillment of objectives, which leads athletes to demotivate, maintaining anxiety for their next competitions.

KEYWORDS: precompetitive anxiety, motivation, swimming, sport psychology.

\section{Resumo:}

O objetivo desta pesquisa é descrever o manejo da ansiedade pré-competitiva em nadadores de elite costarriquenhos entre $14 \mathrm{e}$ 22 anos de idade, identificando os elementos motivadores (externos e internos) e como a relação desses atletas com seu treinador e com seu grupo influencia nos processos de motivação e ansiedade pré-competitiva. Para isso, foram realizadas entrevistas semiestruturadas no final de 2018. Os resultados sugerem que entre os principais elementos associados a maiores sentimentos de ansiedade estão a falta de objetivos alcançados antes da competição, o mau desempenho durante os treinos e a consciência da importância da respetiva competição. Suas motivações foram classificadas em intrínsecas (competição olímpica, melhora de tempo, colocação em rankings) e extrínsecas (família, técnico, equipe). Quando há um relacionamento ruim com o treinador e com seu grupo, existe uma perda de interesse em ir treinar e competir. Concluindo, ao apresentar ansiedade pré-competitiva, o desempenho pode diminuir causando o não cumprimento dos objetivos, o que leva os atletas a começarem a se desmotivar, mantendo a ansiedade para as próximas competições.

PALAVRAS-CHAVE: ansiedade pré-competitiva, motivação, natação, psicologia do esporte. 


\section{INTRODUCCIÓN}

En el deporte existen variables ajenas al ámbito estrictamente físico que influyen en el rendimiento, como es el caso de la motivación, la autoeficacia, la atención, la ansiedad o el estrés (Molina et al., 2013), las cuales forman parte de lo que conocemos como la psicología deportiva, un área en la cual se aplican principios psicológicos a la actividad física y al deporte en todos los niveles de desarrollo humano, a la vez que se tratan de incorporar enfoques y metodologías que son propias de la disciplina psicológica (Browne y Mahoney, 1984).

La ansiedad, según Rosselló et al., (2016), se entiende como un estado psicológico y fisiológico caracterizado por componentes cognoscitivos, somáticos, emocionales y conductuales, los cuales combinados producen nerviosismo, temor o preocupación. Dicho estado de ánimo puede experimentarse de manera generalizada ante distintos estímulos externos que puedan ser percibidos como amenazantes, si bien la presencia de dicho estímulo puede no necesariamente estar presente. Fortes et al, (2017) mencionan que la ansiedad es una construcción multidimensional, en la que interactúan variables cognitivas, fisiológicas y motoras (González et al., 2017); y se relaciona con la voluntad de responder al estrés y de percibir situaciones estresantes (Fernandes et al., 2013).

La ansiedad en el contexto del rendimiento deportivo se entiende como un estado emocional inapropiado, caracterizado por el nerviosismo y la preocupación. Se hace diferencia entre la ansiedad cognitiva, lo que corresponde a preocupaciones que caracterizan el estilo del pensamiento, y la ansiedad somática, en referencia al aspecto orgánico de ese estado emocional (Gutiérrez et al., 2013). Gónzalez et al. (2017) aducen que al manejar niveles altos de ansiedad en el deporte se reduce el rendimiento, en el tanto afecta la calidad de la atención y, como consecuencia, la ejecución. Wolanin et al. (2015) y Kendall y Hollon (2013) reportan niveles de ansiedad significativamente distintos para atletas respecto al resto de la población, lo cual podría explicarse debido al hecho de que estos últimos conviven con una fuerte presión que es propia de las diferentes actividades a las cuales están expuestos (entrenamientos, competencias, etc.); un aspecto que a su vez estaría relacionado con una serie de condicionantes históricos, económicos y socioculturales (Calero y Suárez, 2011).

Desde la teoría de la autodeterminación, se entiende la motivación como un continuo caracterizado por tres grandes componentes: una motivación intrínseca (se involucra en una actividad por su propia iniciativa, desea cumplir sus propios objetivos por placer y satisfacción de sí mismo), una motivación extrínseca (como un medio para conseguir un fin y no por su propio beneficio) y la amotivación (la condición en la cual no hay motivación) (Zarauz y Ruiz-Juan, 2014). A la vez, propone que los individuos tienen múltiples motivos, los cuales pueden diferenciarse por su grado de internalización o autodeterminación; cuanto más internalizado sea el motivo, más autónoma será la persona cuando actúe (Guzmán y Kingston, 2013).

Por otra parte, Zarauz y Ruiz-Juan (2014) mencionan que la motivación hace referencia a cómo interactúan las variables sociales, ambientales e individuales, determinando la elección de una u otra actividad deportiva, la intensidad de su entrenamiento, la persistencia y el rendimiento en ella. Botvinick y Braver (2015) conceptualizan a la motivación como un elemento orientador y estimulante de la recompensa intrínseca y extrínseca, tanto en lo cognitivo como en lo conductual. Ambos autores sostienen que el contendido motivacional es además específico, en el tanto las personas tienden a esforzarse más por los objetivos que encuentren más gratificantes (Botvinick y Braver, 2015).

De esta manera, la motivación resulta un aspecto sustancial en atletismo, ya que determina la elección de una u otra actividad deportiva, la intensidad de la práctica, la persistencia de la tarea y del rendimiento en esta misma, lo cual previene a su vez una serie de situaciones como la ausencia de objetivos, los sentimientos depresivos o la incompetencia, que al mismo tiempo podrían contribuir a la ansiedad (Zarauz y Ruiz-Juan, 2014).

Un aspecto no menos importante dentro de la motivación consiste en la calidad de la relación que sostenga el sujeto deportista con su entrenador o entrenadora, ya que este es el principal responsable de enseñar las habilidades físicas, técnicas y tácticas que permiten sacar el máximo potencial del rendimiento de la 
persona, así como de procurar su crecimiento fuera del campo deportivo. Caso contrario, la ansiedad y la desmotivación en los entrenamientos y competencias constituiría una consecuencia probable (Rodríguez et al., 2017).

Por otra parte, quienes integran un determinado equipo se vinculan a través de sus intereses, aspiraciones comunes para lograr una meta o el objetivo propuesto, y se ha comprobado que, si se cumple, provoca un estado de satisfacción muy favorable en cada integrante (Garimaldi, 2016). En el caso particular de la natación, en algunas ocasiones puede suceder que sus pares se conviertan en sus oponentes, ya que podrían existir varios integrantes del mismo grupo que compitan en categorías y pruebas similares. Esto último podría de igual forma generar ansiedad precompetitiva, con pensamientos relacionados a la superioridad del otro sujeto, aunado a otras preocupaciones que afectarían el rendimiento de manera negativa (Jorgge, 2013).

A pesar del hecho de que la natación es una disciplina individual, existen factores del entorno social deportivo que resultan de igual forma importantes, como la solidaridad, la cohesión de grupo, el compañerismo y la aceptación dentro del equipo, los cuales contribuyen a que el individuo pueda sentirse seguro, cómodo y apoyado en su entrenamiento o que, por el contrario, se sienta fuera de dicha agrupación y ansioso (Paredes et al., 2016). En este sentido, los autores señalan también la existencia de ansiedad en situaciones en que los deportistas puedan sentirse muy evaluados en cuanto a si están logrando la meta.

La natación se caracteriza por esfuerzos continuos y cíclicos de alta intensidad, en la que la competencia constituye un factor estresante para atletas. Lo normal es que un sujeto nadador compita entre dos a cuatro pruebas por torneo. Según la Federation International de Natation (2016), los eventos oficiales de natación son 50 metros libre, 100 y 200 metros libre, pecho, dorso y mariposa, 400 libre y combinado y 1500 libre. Cuando un nadador o una nadadora se encuentra esperando en el panel de control antes de la competencia, puede empezar a percibir cierta ansiedad; por ejemplo, ante el contacto visual con sus oponentes o al escuchar su nombre mencionarse a través de los parlantes (Fortes et al., 2017).

Diversas investigaciones sobre la ansiedad en la psicología deportiva han mostrado que la presencia de ansiedad en situaciones de presión conduce a un deterioro en la ejecución de los procesos habituales del rendimiento (Molina et al., 2013). En este sentido se utiliza el término "choking under pressure", un fenómeno en el que la presión se define como una combinación de factores que incrementan la demanda de la situación, mientras que el choking designa a la reducción del rendimiento bajo las situaciones de presión (Baumeister, 1984).

Evidentemente, en las competiciones deportivas hay siempre presión, lo que implica estados emocionales como el nerviosismo o la preocupación que conllevan a un fallo en los procesos atencionales, la toma de decisiones y el control emocional (Fortes et al., 2017). También, Fortes et al. (2017) encuentran relaciones significativas entre la ansiedad cognitiva, la ansiedad somática y el ritmo cardíaco, lo que indica que los atletas con una mayor ansiedad cognitiva y/o somática presentan una alteración del sistema nervioso. Por el contrario, si el individuo presenta condiciones afectivas positivas, entre ellas la alegría (Gutiérrez et al., 2013) o un perfil resiliente alto y optimista, es en mayor medida capaz de soportar niveles de carga y de estrés dentro del entrenamiento, con lo que podría tener una mejora en su rendimiento competitivo (Aranzana et al., 2018).

En un estudio donde analizaban la ansiedad precompetitiva en nadadores brasileños encontraron diferencias entre ansiedad cognitiva y ansiedad somática, indicando que los nadadores masculinos tienen un perfil más seguro de sí mismo y se sienten menos preocupados, presentando menos pensamientos negativos en momentos previos a la competencia. Asimismo, demuestran que los que participan en una mejor clasificación experimentan menos ansiedad que los que compiten en un menor nivel competitivo (Branco et.al, 2019).

Por otra parte, en una investigación que exploró las diferencias entre el waterpolo y la natación sobre la ansiedad precompetitiva, las estrategias de afrontamiento y la relación entre ambas, concluyen que los deportes de equipo proporcionan mayor confianza comparados con las prácticas individuales, lo que supone que en natación se sufre una mayor ansiedad cognitiva y somática que en waterpolo (Sepúlveda et al., 2019). 
Pineda-Espejel et al. (2020) descubren en su estudio que deportistas, que se desmotivan, pueden sentir inseguridad, creando sentimientos de tensión, pensamientos negativos debido a la incertidumbre de sus resultados y esto puede llevarles a sentir ansiedad. También, dentro de los resultados destacan que el estilo de apoyo de quien les entrena predice positivamente la autoconfianza antes de una competencia, disminuyendo las ansiedades cognitivas y somáticas y motivándoles más. En cambio, si el entrenador o entrenadora se encuentra gritando, haciendo comparaciones, participan en su deporte por presiones internas o externas, por lo que disminuyen la motivación y aumentan la ansiedad. Por último, destacan que las relaciones interpersonales influyen en la motivación de deportistas.

Por último, no se han encontrado estudios sobre el rendimiento de los sujetos nadadores en Costa Rica ni investigaciones que relacionen la ansiedad precompetitiva con la natación. Es por esta razón que la presente investigación se propone el explorar, de forma cualitativa e inicial, el manejo de la ansiedad precompetitiva en nadadores y nadadoras de élite costarricenses, identificando los elementos motivacionales (externos e internos), así como explorando la relación de dichos sujetos atletas con la persona que les entrena y con su grupo de pares, todo lo cual permitirá no solo realizar intervenciones psicológicas dirigidas a mejorar el rendimiento competitivo, sino también su calidad de vida.

\section{Metodología}

La presente investigación tiene un enfoque cualitativo (Mason, 2018), en el que se aplicó una entrevista semiestructurada. El alcance es de tipo descriptivo (Hernández et al., 2014), que pretende especificar las propiedades, características y perfiles de las personas, grupos, comunidades entre otros datos sometidos a un análisis. En este sentido, la investigación se dirige hacia describir cómo se maneja la ansiedad precompetitiva en nadadores y nadadoras que se encuentran practicando y compitiendo la disciplina de manera activa.

\section{Participantes}

En esta investigación se planea evaluar a siete jóvenes nadadores costarricenses, cuatro participantes femeninos y tres masculinos, de quienes dos se encuentran en la categoría Juvenil A (13 a 14 años) y cinco en Mayor (18 y más) (Federación Costarricense de Deportes Acuáticos, 2018). Adicionalmente, para poder efectuar la evaluación, se ha determinado que deben continuar entrenando, compitiendo activamente y estar en los primeros lugares del ránking nacional. Los sujetos participantes indican que llevan compitiendo desde que iniciaron la categoría infantil (si bien algunos compiten desde 6 y 8 años) y que entrenan dos horas diarias entre 5 y 6 días a la semana.

Los participantes fueron debidamente informados de la investigación y del carácter voluntario de la misma a través de la entrega de un consentimiento informado, mediante el cual se les solicita a estos y a sus padres/ madres de familia (en el caso de atletas menores de edad) su consentimiento y disposición para ser parte de la investigación, garantizándoles a su vez el anonimato de los datos proveídos.

Adicionalmente, el consentimiento informado y la investigación contaron con un aval del comité evaluador de la Universidad de Iberoamérica para su implementación.

\section{Instrumentos}

Se utilizó la entrevista semiestructurada, la cual consiste en una guía de preguntas; sin embargo, el entrevistador tiene la libertad de introducir otras interrogantes adicionales para obtener más información o aclarar exhaustivamente el objetivo de la interrogación (Hernández et al., 2014). 


\section{Procedimiento}

Respecto a la recolección de datos, primeramente, se procedió a elegir los sujetos nadadores que se encuentran dentro los primeros lugares del ránking nacional y luego se les contactó a través de sus redes sociales o número de teléfono, donde se les explicó sobre el trabajo de investigación y sobre la intención de realizarles una serie de preguntas sobre ansiedad, motivación, relación con el entrenador o entrenadora y su grupo de pares y así luego aprobarían su participación en el estudio.

Posteriormente se realizó una entrevista semiestructurada, para la cual la entrevistadora contaba previamente con las interrogantes sobre el tema que se les plantearía a todo el grupo de participantes en algunas entrevistas se tuvo la oportunidad de hacer otras preguntas-. A cada participante se le hizo la entrevista en línea, ya que a la totalidad se le contactó por su número de teléfono o sus redes sociales y sus respuestas quedaron escritas. Esta entrevista consta de nueve preguntas abiertas (Ver Anexo 1), en la que todos los sujetos participantes pueden expresar lo que sienten y piensan antes de las competencias, cómo logran manejar su ansiedad en momentos previos a la competencia, cuáles son sus motivaciones y la importancia de tener una buena relación con quien les entrena y compañeros y compañeras.

\section{Técnica de análisis}

En dicho apartado, se implementó un análisis de contenido cualitativo de tipo reductivo (Mayring, 2004). A partir de las preguntas de investigación, se tomaron las temáticas básicas para la definición de categorías y subcategorías en base a los antecedentes teóricos. De este modo, se especificaron las categorías que parten del examen de un subconjunto de datos recolectados, y estas a su vez fueron corregidas posteriormente, para analizarlas nuevamente dentro del conjunto del material.

\section{Resultados}

Dentro del análisis de resultados se escogieron diferentes categorías atendiendo los dos temas principales: ansiedad precompetitiva y motivación. En cada categoría se discriminó una serie de subcategorías respondiendo a indicadores aportados por los distintos entrevistados y vinculados con la teoría mencionada anteriormente. En la tabla 1 se detalla y permite manejar de una mejor manera la información recogida de las entrevistas.

TABLA 1

Sistema de categorias y subcategorias

\begin{tabular}{lll}
\hline \multicolumn{2}{c}{ Categoría } & \multicolumn{1}{c}{ Subcategorías } \\
\hline 1. Ansiedad precompetitiva & $\bullet$ & Sensaciones antes de una competencia \\
& $\bullet$ & Ansiedad somática y cognitiva \\
& & - Técnicas para disminuir la ansiedad \\
2. Motivación & - Motivación extrínseca \\
& - Motivación intrínseca \\
& - Relación con el entrenador \\
& &
\end{tabular}


Además, dentro de la presentación de los resultados e interpretación de las entrevistas, se hará un análisis desde la perspectiva de las diferentes categorías, profundizando en las subcategorías definidas tal como se especifican en las tablas 2 y 3.

TABLA 2

Definiciones de subcategorias de ansiedad precompetitiva

\begin{tabular}{lll}
\hline \multicolumn{1}{c}{ Ansiedad precompetitiva } \\
\hline 1. Sensaciones antes de una competencia & $\begin{array}{l}\text { Definición } \\
\text { Es lo que el nadador o la nadadora percibe en su cuerpo } \\
\text { y mente antes de iniciar su competencia. } \\
\text { 2. Ansiedad somática y cognitiva }\end{array}$ & $\begin{array}{l}\text { Identificán de síntomas que siente en su cuerpo } \\
\text { o qué pensamientos aparecen antes y durante de la } \\
\text { competición. }\end{array}$ \\
& $\begin{array}{l}\text { Qué elementos utiliza para disminuir la ansiedad } \\
\text { precompetitiva. }\end{array}$ \\
\hline
\end{tabular}

TABLA 3

Definiciones de subcategorias de motivación

\begin{tabular}{|c|c|}
\hline \multicolumn{2}{|c|}{ Motivación } \\
\hline Subcategoría & Definición \\
\hline 1. Motivación intrínseca & $\begin{array}{l}\text { Estímulos internos que tiene el nadador para estar } \\
\text { participando en el deporte de la natación y en } \\
\text { competiciones de alto rendimiento. }\end{array}$ \\
\hline 2. Motivación extrínseca & $\begin{array}{l}\text { Incentivos o alicientes externos que lo ayudan a seguir } \\
\text { compitiendo. }\end{array}$ \\
\hline 3. Relación con el entrenador & $\begin{array}{l}\text { La importancia de mantener una buena relación con el } \\
\text { entrenador para seguir motivado. }\end{array}$ \\
\hline 4. Relación con el grupo de pares & $\begin{array}{l}\text { Consideraciones respecto a la calidad de las relaciones } \\
\text { con el grupo de pares y cómo ayuda a que los atletas } \\
\text { se mantengan motivados en la práctica del deporte y } \\
\text { dentro de las competiciones. }\end{array}$ \\
\hline
\end{tabular}

En cuanto a lo referido por el grupo de atletas propiamente sobre las sensaciones antes de una competencia, se puede evidenciar que, dependiendo del proceso de preparación que hayan llevado antes de la competencia y la trascendencia de esta, pueden sentir algún nivel de ansiedad, como mencionan algunos sujetos participantes.

Ejemplo 1, participante 5

"Varía dependiendo de qué tan buena o qué tan mala haya sido mi preparación. Si había venido entrenando bien, voy a estar muy positivo; nervios, pero pocos y sabiendo que posiblemente voy a mejorar. Pero si la preparación previa no ha sido la mejor, puede que esté con pensamientos negativos y con miedo a que me vaya mal".

También expresan que la prueba específica en que vayan a competir los puede poner con más ansiedad. Ejemplo 2, participante 2 
“ $i$ Todo depende de cada competencia y depende de qué tan importante sea! También depende de la prueba que vaya a competir, porque hay ciertas pruebas en las que me pongo más nerviosa que para otras. Siento que se me va el aire y me pongo ansiosa".

Asimismo, cabe mencionar que, de acuerdo con el grado de la importancia que perciban de la competencia, muchos de los síntomas descritos antes de la misma consisten en la falta de aire, náuseas, pensamientos negativos, elevación de la frecuencia cardíaca, tensión muscular, temblor en las manos, miedo a perder, entre otros. Los sujetos designan la anterior sintomatología con la etiqueta de "ansiedad" o "nervios".

Ejemplo 3, participante 3

"Si es un torneo muy importante me dan ganas de vomitar, me desconcentro mucho".

\section{Ejemplo 4, participante 6}

"Normalmente antes de una competencia importante me siento muy ansiosa; la frecuencia cardiaca se me eleva bastante. Cuando es mucha presión y no lo logro controlar, me pueden dar ganas de vomitar y mucha tensión”.

Del mismo modo, se podría pensar que los sujetos participantes experimentan una serie de temores mientras esperan su competencia, lo cual posibilita el aumento de su ansiedad debido a la presión. Algunos de estos temores se dirigen a que sus músculos no puedan reaccionar, a que la competición les salga mal, a equivocarse y al fallo en sus objetivos; pongamos por caso el hecho de no lograr los tiempos.

Ejemplo 6, participante 3

"Me preocupo de no equivocarme en hacer lo que mi entrenador me diga; en bajar el tiempo".

\section{Ejemplo 7, participante 6}

"Siempre puedo tener el pensamiento de que me da miedo fallar para lo que me he preparado".

Ahora bien, la mayoría indica que no les cuesta concentrarse y son personas que se enfocan mucho en su táctica para la prueba.

Ejemplo 9, participante 2

"Me enfoco en cómo tengo que nadar la prueba y en que quiero ganar".

Sin embargo, algunos sujetos mencionan que depende mucho de la relevancia del evento, la preparación o de su estado de ánimo, debido a la presión.

Ejemplo 10, participante 5

"Dependiendo del nivel de la competencia. Si es una competencia muy importante, tipo CAMEX, CCCAN, Mundial, no. Si es algún fogueo o alguna competencia no tan importante, se me facilita más la concentración”.

\section{Ejemplo 11, participante 3}

"A veces, depende de demasiadas cosas, como qué tan preparada me sienta, qué prueba vaya a nadar, mi ánimo ese día".

Podemos, de igual forma, apreciar a continuación una serie de técnicas diferentes para disminuir la ansiedad.

Ejemplo 13, participante 6

"La visualización y técnicas de respiración".

Ejemplo 14, participante 2

"Respirar profundo y controlar mi respiración, moverme y tirarme agua antes de tirarme a competir". 
Otro de los factores fundamentales concierne a la motivación. La totalidad llevan más de nueve años practicando este deporte, debido a que les gusta hacer deporte en general y coinciden en que la natación es un deporte completo.

Ejemplo 16, participante 2

"Nado porque me gusta el deporte en sí, me gusta competir y me gusta que sea individual y que el esfuerzo para mis resultados solo depende de mí".

También, en la información recolectada se obtiene que el grupo de atletas exterioriza los deseos de superarse a sí, como el hecho de que el esfuerzo que hagan depende de sus propias personas, lo cual demuestra que se tienen motivación para superarse cada día y para sentirse bien y alegres con los resultados (Figura 1).

Ejemplo 17, participante 5

"Lo practico porque me apasiona muchísimo. Me apasiona muchísimo no el hecho de entrenar y prepararme, sino más que todo el hecho de ir a una competencia y lograr superarme a mí mismo/a, lograr bajar mis tiempos y, si se puede, ganar”.

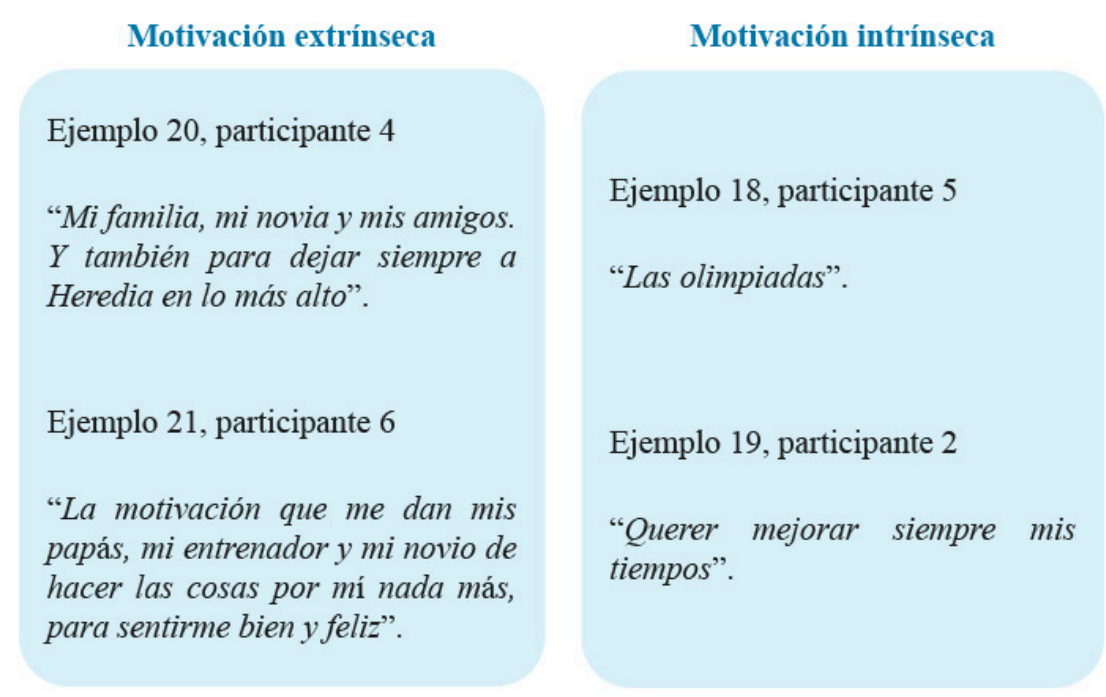

\section{FIGURA 1}

Motivaciones

De la misma manera, según lo expresado anteriormente, se habla del entrenador o entrenadora como una de las motivaciones para deportistas, ya que es uno de los individuos que pasa la mayor parte del tiempo con él o ella y es alguien que conoce cada aspecto (habilidades, debilidades) de sus atletas, así como alguien que sabe cómo deben entrenar con la finalidad de conseguir los objetivos.

Por último, la relación entre pares de las agrupaciones parece ser otro factor, ya que algunos sujetos manifiestan que esto puede afectarles en su motivación porque se pueden sentir excluidos (Figura 2). Sin embargo, existen otros que piensan que no les afecta, ya que algunos entrenan independientemente o que hay cooperación dentro de los equipos, sobre todo entre amistades cercanas. En cambio, cuando se encuentran en la selección nacional revelan que las relaciones entre pares no son tan cercanas y que no hay tanto apoyo. 
Relación con el entrenador/a

Ejemplo 22, participante 5

"Totalmente sí. Porque no me imagino un entrenamiento súper duro, donde el entrenador no lo comprenda a uno o donde no lo trate como se debe. Al menos yo con mi carácter, si me tratara mal, no lograría estar en el nivel que estoy".

Ejemplo 23, participante 2

" iSí, porque él es el que lo entrena a uno y el que eventualmente antes de una selección nacional va a velar por uno! Si hay una mala relación el entrenador ni siquiera va a querer entrenarme".
Relación con el grupo de pares

Ejemplo 24, participante 7

"Si, porque uno se va a sentir solo y no va a haber interés".

Ejemplo 25, participante 5

"Yo estoy prácticamente entrenando solo... Pero no es algo que me afecte porque realmente me gusta mucho entrenar solo".

Ejemplo 28, participante 6

"Entre compañeros de equipo sí, pero entre compañeros de selección es con algunos solamente".

\section{FIGURA 2}

Relaciones con quien les entrena o con sus pares

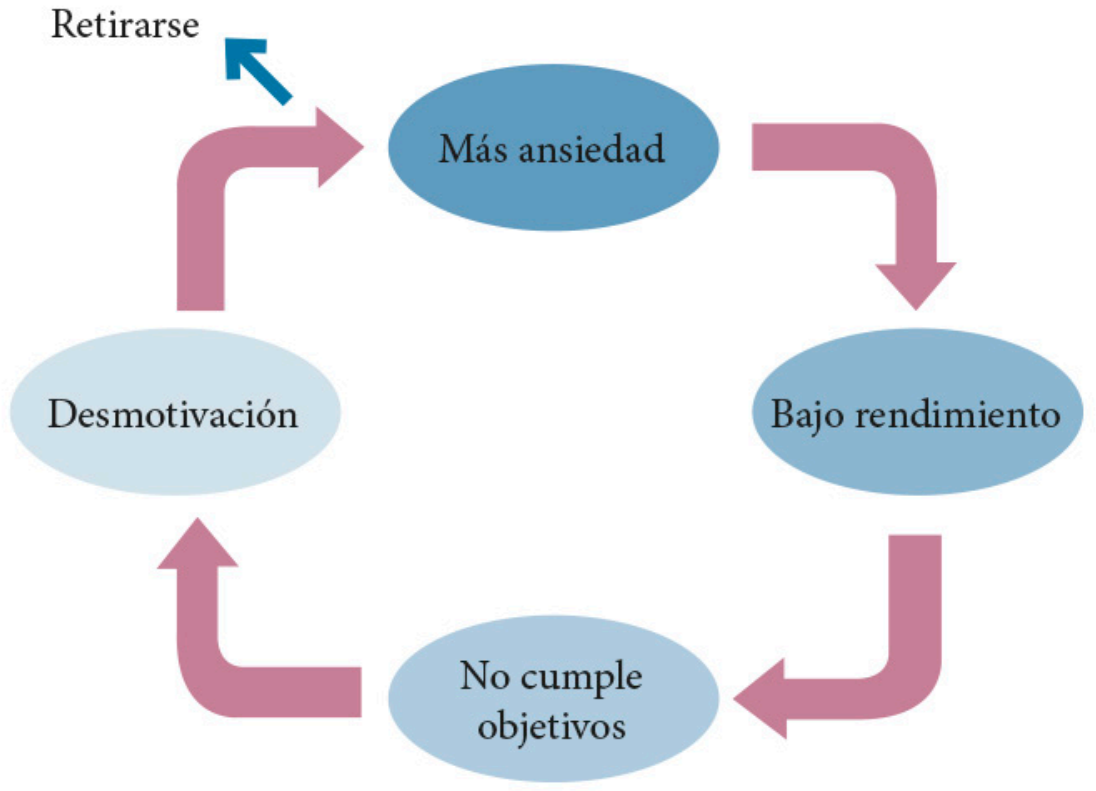

FIGURA 3

Ciclo de ansiedad precompetitiva. 


\section{Discusión}

El objetivo de esta investigación era describir cómo se maneja la ansiedad precompetitiva en los grupos nadadores que se encuentren entrenando y compitiendo activamente, mediante identificar los elementos motivantes y el cómo influye la relación de dichos sujetos atletas con su entrenador o entrenadora y con su grupo de pares en los procesos de motivación y ansiedad precompetitiva (Figura 3). Los resultados sugieren que, dependiendo de la preparación y la trascendencia del certamen, se puede presenciar un cierto nivel de ansiedad. En otras palabras, si se trata de una competición internacional o alguna competencia nacional para establecer marcas, el grado de ansiedad podría elevarse. Sobre este punto, Branco, Leal et al. en un estudio del 2019 encuentran precisamente lo contrario: quienes participan en procesos de clasificación mayor presentaron menos ansiedad respecto a quienes indican un nivel menos competitivo. De esta manera se evidencia la necesidad de estudiar este aspecto desde una perspectiva cuantitativa y tomando en cuenta distintas variables transculturales subyacentes.

Resulta además interesante la importancia que adquiere la preparación previa para atletas, en el tanto estos tienen la creencia de que, si la misma es de calidad, se alcanzarán los objetivos. Buceta (1998) muestra en un estudio previo que existen ciertas causas que pueden producir ansiedad, dependientes de la sustancialidad del evento; por ejemplo, la exigencia de lograr ciertos objetivos en algunas competencias, o la tensión de estar representando a un país.

Asimismo, otras investigaciones hablan acerca de la presión, la cual funciona como un indicador que incrementa debido a diferentes factores durante una situación, como en el caso concreto de los deportistas que buscan superar al rival o a sí. Dicha competencia se puede evidenciar en sus resultados; motivo por el cual, al experimentar estas presiones psicológicas, se obtiene la afectación de sus objetivos en la competición y del rendimiento de cada deportista en consecuencia (Molina et al., 2013).

A la luz de la teoría multidimensional de la ansiedad precompetitiva (Martens et al., 1990) se evidencia principalmente la falta de aire, náuseas, elevación de la frecuencia cardíaca, tensión muscular y el temblor en las manos como manifestaciones somáticas; así como una serie de pensamientos negativos experimentados primordialmente a través del miedo a perder o de la preocupación respecto a que los músculos no reaccionen dentro del componente cognitivo.

Por otro lado, en cuanto a estrategias de afrontamiento para controlar la ansiedad, los sujetos participantes mencionaron la respiración, la visualización, el darse a sí mismos motivación, o el distraerse con otras actividades que no tengan relación con la competencia, como escuchar música.

Dichas tácticas según Paredes et al. (2016), son estrategias de afrontamiento fundamentales para deportistas, que permiten controlar la ansiedad, ayudar a proyectar seguridad, aumentar la concentración, aprovechar oportunidades, entre otros.

Del mismo modo, se ha observado en el estudio de Galletebeitia (2015) que deportistas antes de competir pueden llegar a percibir un ambiente estresante que les genera ansiedad. Como resultado, se corre con la posibilidad de experimentar desconcentración, dificultades en el control del pensamiento, dificultades motivacionales y disminución de su autoconfianza. Como lo indican Sepúlveda et al. (2020), los sujetos nadadores al ser un deporte individual proporcionan menor autoconfianza y mayor ansiedad cognitiva y somática. Sin embargo, dentro de los sujetos entrevistados se pudo identificar que la mayoría manifiesta no perder la concentración; por el contrario, intentan siempre estar enfocados en la prueba de natación y en cómo la ejecutarán (Galletebeitia, 2015).

Ahora bien, la natación parece ser practicada por los sujetos participantes desde una motivación intrínseca, en el tanto argumentan que es un ejercicio completo, y que les gusta superarse en el sentido de que el esfuerzo emprendido depende de ellos mismos. Estos aspectos indican que realizan la actividad por su propia iniciativa en el alcance del deleite y satisfacción por la participación y lograr ciertos objetivos cuya intención es mejorar los tiempos, clasificar a los Juegos Olímpicos, entre otros. 
Lo extrínseco en la motivación a la práctica parece encontrarse sobre todo en el apoyo hacia la consecución de un fin, sea este satisfacer a la familia o a uno de los sujetos esenciales para un nadador o nadadora, como lo es la persona entrenadora, con quien cada deportista pasa jornadas extensas y que es quien conoce cada aspecto de la preparación, siendo a su vez responsable de enseñar las habilidades físicas, técnicas y tácticas que permiten sacar el máximo potencial de sus atletas (Rodríguez et al., 2017). Sin embargo, en los casos profundizados podemos encontrar que, con una mala relación con quien les entrena, se pierde también el interés de ir a entrenar y competir, tal como fue encontrado por Rodríguez et al. (2017), en el sentido de que cuando no se fomenta una interacción abierta y sincera, pueden aparecer muchos inconvenientes en los entrenamientos y competencias, entre ellos la ansiedad y desmotivación por la falta de apoyo y confianza. Asimismo, Pineda-Espejel et al. (2020) refuerzan lo explicado anteriormente, indicando que un entrenador que grite o compare hace que sus atletas participen teniendo alguna presión, y provoca la disminución de su motivación y un aumento en la ansiedad.

Por último, otro de los factores que influyen dentro de la ansiedad y motivación es la relación de pares, ya que confiesan que la ausencia de un buen vínculo provoca el sentimiento de la soledad, lo cual conlleva a la afectación en el nivel de motivación. Además, comentan que existe una buena conexión solo con los individuos considerados como amistades cercanas; en cambio, si se encuentran en la selección nacional la afinidad no es tan cercana. Por consiguiente, se podría revelar, según estudios (Garimaldi, 2016; Jorgge, 2013), que, al ser algunos sujetos de la misma categoría, se vuelven oponentes en esta prueba, de forma que podría generar ansiedad precompetitiva debido a los pensamientos negativos consiguientes, con el consiguiente distanciamiento en la relación (Jorgge, 2013).

\section{Conclusiones}

Se encuentra que la ansiedad reportada por los sujetos participantes se explica por diferentes situaciones, ya sea por una competición importante o por no haber tenido una buena preparación en los entrenamientos. Esta ansiedad se puede entender ya sea desde un aspecto cognitivo o somático (Gutiérrez et al., 2013), existiendo un grado de variación en los síntomas experimentados entre unos y otros que se ponen de manifiesto sobre todo en pensamientos de que no se encuentran preparados para la prueba. Sin embargo, muchos de ellos y ellas mencionan que aplican diferentes técnicas de afrontamiento para disminuir la ansiedad, tales como la visualización, diferentes tipos de respiración y el motivarse a sí mismos con palabras.

En segundo lugar, la motivación es un factor que entra en juego en dicha disciplina, debido a que si existe desmotivación el rendimiento también bajará y producirá ansiedad, ya que no alcanzarán sus metas como lo desean. Por eso, como lo indican las personas entrevistadas, es primordial que el sistema social detectado alrededor de su entorno diario en los entrenamientos y competencias (es decir, los entrenadores o entrenadoras) realice un balance para la creación de un buen ambiente en el equipo con cada integrante. Ese equilibrio se debe formar con que el sujeto entrenador mantenga una buena comunicación con sus atletas y demuestre confianza, todo con el fin de estar motivándoles a cumplir sus objetivos.

Otro aspecto destacable con base en la información obtenida en esta investigación concierne a que, debido a las presiones internas por lograr los objetivos de resultados y de tarea, por ejemplo, ir a las olimpiadas o mejorar sus tiempos, los grupos de atletas podrían presentar ansiedad precompetitiva, lo cual facilita el proceso de creación del ciclo de ansiedad. Por lo que es esencial que quienes practican la natación y todas las personas deportistas de diferentes modalidades tengan presente el factor psicológico dentro de la preparación integral, en el que puedan aprender el modo de autocontrolarse, sobre su estabilidad emocional, concentración, técnicas de relajación, su motivación para mejorar su rendimiento y sentir seguridad antes de una competencia (Linares, 2016).

Finalmente, la conexión entre pares parece ser un elemento para tener muy en cuenta, dada la importante influencia que podría ejercer en la motivación de cada sujeto, de manera que, si no existe un buen vínculo, los 
atletas o las atletas podrían sentir les rechazan y el ambiente volverse negativo. Debido a que en ocasiones los sujetos nadadores se encuentran en la misma categoría competitiva, cabe la posibilidad de que se establezca una rivalidad, lo cual se destaca mucho en los deportes individuales, provocando un aumento en la ansiedad así como en pensamientos negativos surgidos de su propia presión. En caso de reaparecer el ciclo de ansiedad, el resultado final puede ser que el individuo baje rendimiento o, aún peor, se vea empujado al retiro.

\section{REFERENCIAS}

Aranzana, M., Salguero, A., Molinero, O., Boleto, A.F. y Márquez, S. (2018). Relación de la carga interna de entrenamiento, optimismo y resiliencia con los niveles de estrés-recuperación en nadadores. Cuadernos de Psicologia del Deporte, 18(1), 43-54. https://search.proquest.com/openview/e32919bbcela35dbbaee5cf9904a 5ec4/1 ?pq-origsite $=$ gscholar\&cbl $=2035768$

Baumeister, R. F. (1984). Choking under pressure: self-consciousness and paradoxical effects of incentives on skillful performance. Journal of Personality and Social Psychology, 46(3), 610-620. https://doi.org/10.1037//0022-351 4.46.3.610

Botvinick, M., \& Braver, T. (2015). Motivation and Cognitive Control: From Behavior to Neural Mechanism. Annual Reviews of Psychology, 66, 83-113. https://doi.org/10.1146/annurev-psych-010814-015044

Branco, G. C., Leal, A. C., Andrade, J. R., Laranjeira, C. T., Quinta, E. F. y Ferreira, G. (2019). Analysis of precompetitive anxiety of Brazilian young swimmers. Acta Scientiarum. Health Sciences, 41. https://doi.org/10.40 25/actascihealthsci.v41i1.45475

Browne, M. A., \& Mahoney, M. .J. (1984). Sport Psychology. Annual Reviews of Psychology, 35, 605-625. https://ww w.annualreviews.org/doi/pdf/10.1146/annurev.ps.35.020184.003133

Buceta, J. M. (1998). Psicología del entrenamiento deportivo. Dykinson.

Calero, S. y Suárez, C. (2011). Acciones para perfeccionar la selección de talentos del voleibol en los programas cubanos de deporte escolar. Lecturas: Educación Física y Deportes, 16(156), 1-7. https://www.efdeportes.com/efd156/p erfeccionar-la-seleccion-de-talentos-del-voleibol.htm

Federación Costarricense de Deportes Acuáticos (FECODA). (2018). Natación. https://www.fecodacrc.org/discipl inas/natacion/

Federation Internationale de Natation (2016). URL: http://www.fina.org/

Fernandes, M. G., Nunes, A. S., Vasconcelos-Raposo, J., Fernandes, H. M., \& Brustad, R. (2013). The CSAI-2: An examination of the instrument's factorial validity and reliability of the intensity, direction and frequency dimensions with Brazilian athletes. Journal of Applied Sport Psychology, 25(4), 377-391. https://doi.org/10.10 80/10413200.2012.744780

Fortes, L.S., da Costa, B., Paes, P.P., do Nascimento, J., Fiorese, L., \& Ferreira, M. (2017). Influence of competitiveanxiety on heart rate variability in swimmers. Journal of Sports Science and Medicine, 16(4), 498-504. https://w ww.ncbi.nlm.nih.gov/pmc/articles/PMC5721179/

Galletebeitia, L. (2015). La dureza de personalidad en las modalidades de fondo y su influencia en la calidad de vida del deportista. [Tesis de maestría]. Facultad de Ciencias de la Actividad Física y del Deporte. Universidad de León. https://buleria.unileon.es/handle/10612/5393

Garimaldi, H.R. (2016). Los seres del tiempo: La permanencia del deportista en la natación competitiva. [Tesis de posgrado]. Universidad Nacional de La Plata. http://www.memoria.fahce.unlp.edu.ar/tesis/te.1248/te.1248.p $\mathrm{df}$

González, G., Valdivia-Moral, P., Cachón, J., Zurita, F. y Romero, O. (2017). Influencia del control del estrés en el rendimiento deportivo: la autoconfianza, la ansiedad y la concentración de deportistas. RETOS. Nuevas tendencias en Educación Física, Deporte y Recreación, 32, 3-6. https://www.redalyc.org/pdf/3457/3457511000 01.pdf 
Gutiérrez, J. C., Gutiérrez, R. y Ureña, P. (2013). Autoeficacia general, ansiedad precompetitiva y sensación de fluir en jugadores(as) de balonmano de la selección nacional de Costa Rica. MHSalud: Revista en Ciencias del Movimiento Humano y Salud, 10(2). 1-16. http://www.redalyc.org/articulo.oa?id=237029450002

Guzmán, J. F. \& Kingston, K. (2013). Coaches' need satisfaction and self-determination predict perceived promotion of athletes' well-being. Wulfenia, 20(4), 107-120.

Hernández, R., Fernández, C. y Baptista, P. (2014) Metodología de la investigación ( $6{ }^{\text {ta }}$ ed). McGraw-Hill.

Jorgge, Z. M. (2013). Estudio de la ansiedad en los deportistas de la disciplina de atletismo, entre 16 y 19 años de edad, de la Federación Deportiva del Guayas. [Tesis de grado]. Universidad de Guayaquil. http://repositorio.ug.edu.e c/handle/redug/5865

Kendall P. C. \& Hollon, S. D. (2013). Cognitive-behavioral interventions: Theory, research, and procedures (7th ed). United States: Academic Press.

Linares, R. E. (2016). Psicología en el deporte: herramientas, metodología y técnicas para mejorar el rendimiento (1.a ed). Editorial Brujas.

Martens, R., Vealey, R. S. \& Burton, D. (1990). Competitive anxiety in sport. Champaign, IL: Human Kinetics.

Mason, J. (2018). Qualitative Researching. (3a ed). SAGE Publications.

Mayring, P. (2004). Qualitative Content Analysis. En U. Flick, E. von Kardorff \& I Steinke (Eds.) A companion qualitative research. (pp.266-269) Sage Publications.

Molina, J., Sandín, B. y Chorot, P. (2013). Sensibilidad a la ansiedad y presión psicológica: Efectos sobre el rendimiento deportivo en adolescentes.Cuadernos de Psicología del Deporte, 14(1) $45-54 \mathrm{http}: / / \mathrm{scielo}$.isciii.es/pdf/cpd/v14 n1/art05.pdf

Paredes, V., Rodríguez, I. y Lira, J. (2016). Intervención cognitivo-conductual para el manejo de ansiedad precompetitiva en nadadores adolescentes. Enseñanza e Investigación en Psicologia, 19(1), 165-177. https://ww w.redalyc.org/pdf/292/29232614011.pdf

Pineda-Espejel, H. A., Morquecho-Sánchez, R. y Alarcón, E. (2020). Estilo interpersonal del entrenador, competencia, motivación y ansiedad precompetitiva en deportistas de alto rendimiento. Cuadernos de Psicologï\#a del Deporte, 20(1), 10-24. http://dx.doi.org/10.6018/cpd.397001

Rodríguez, M. P., Cruz, J. y Torregrosa, M. (2017). Programa de intervención con entrenadores y padres de familia: Efectos en las conductas del entrenador y el clima motivacional del equipo. Revista de la Psicología del Deporte, 26(2), 181-187. https://ddd.uab.cat/pub/revpsidep/revpsidep_a2017v26sup2/revpsidep_a2017v26sup2p181 .pdf

Rosselló, J., Zayas, G. y Lora, V. (2016). Impacto de un adiestramiento en meditación en consciencia plena (mindfulness) en medidas de ansiedad, depresión, ira y estrés y consciencia plena: Un estudio piloto. Revista Puertorriqueña de Psicologia, 27(1), 62-78. https://www.redalyc.org/pdf/2332/233245623005.pdf

Sepúlveda-Páez, G., Díaz-Karmelic, Y. y Ferrer-Urbina, R. (2019). Ansiedad pre-competitiva y estrategias de afrontamiento deportivo, en disciplinas acuáticas individuales y colectivas en deportistas juveniles de alto nivel. Limite (Arica), 14, 1-10. http://dx.doi.org/10.4067/s0718-50652019000100216

Wolanin, A., Gross, M. \& Hong, E. (2015). Depression in athletes: prevalence and risk factors. Current Sports Medicine Reports, 14(1), 56-60. https://doi.org/10.1249/JSR.0000000000000123

Zarauz, A. y Ruiz-Juan, F. (2014) Análisis de la motivación en el atletismo: Un estudio con veteranos. Universitas Psychologica, 13(2), 501-515. https://doi.org/10.11144/Javeriana.UPSY13-2.amae 
MHSALUD, ISSN: 1659-097X, 18(2), Julio-Diciembre, 2021, pP 1-14

HAASE

\section{Anexos}

\section{Entrevista}

Género: F( ) M ( ) Otro ( )

Edad:

Categoría:

1. ¿Por qué practica natación? ¿Hace cuánto la practica?

2. ¿Qué siente antes de las competencias?

3. ¿Cómo podría llamar a esos síntomas que siente?

4. ¿Piensa o siente alguna preocupación antes de competir?

5. ¿Le cuesta concentrarse antes de la competencia?

6. ¿Cuáles son las técnicas que utiliza para disminuir la ansiedad?

7. ¿Es importante para usted tener una buena relación con su entrenador/a? ¿Por qué?

8. ¿Entre sus compañeros/as se dan apoyo?

9. ¿Cuáles son sus motivaciones para lograr sus metas en la natación?

ANEXO 1

Entrevista 